\title{
Ventricular tachycardia with retrograde conduction Simplified diagnostic approach
}

Sir:

In the article by Evans et al., 1974, 36, 512-515) some statements are misleading, and their message, if accepted, will represent a retrogressive step in the diagnosis of arrhythmias. Four of their assertions invite critical comment:

1) 'The diagnosis of ventricular tachycardia is primarily dependent upon the demonstration of dissociated atrial and ventricular activity.'

The urge to find independent atrial activity is a symptom of the 'P-preoccupation syndrome', a disease endemic among cardiographers at all levels of sophistication. For many years those conversant with the diagnosis of arrhythmias have emphasized that AV dissociation is not a valid point in distinguishing ventricular tachycardia from supraventricular tachycardia with aberration. The diagnosis is not 'primarily dependent' on this inadequate clue, and its use is as scientifically acceptable as to assume that the woman on the man's arm is his wife. AV junctional tachycardia with aberration may be dissociated from the atrial rhythm; and ventricular tachycardia may be associated with $1: 1$ retrograde conduction. Neither does dissociated atrial activity prove nor does constantly related atrial activity disprove ventricular tachycardia. Better clues are required and, in recent years, the value of morphological QRS detail has been stressed; certain features, when present, can be of great diagnostic value.

2) "When ventricular tachycardia occurs with intact retrograde conduction the distinction between supraventricular tachycardia with aberrant conduction and ventricular tachycardia with retrograde conduction is considered almost impossible to make.'

If an, at best, inadequate clue- $\mathrm{AV}$ dissociation-is depended upon, obviously diagnosis is rendered impossible by retrograde conduction. But morphological detail, on the other hand, with or without retrograde conduction is equally discriminating. With its help the diagnosis, far from being 'almost impossible', is sometimes extremely easy.

3) 'The presence of a right bundle-branch block pattern, particularly when triphasic, supports a diagnosis of supraventricular tachycardia with aberrant ventricular conduction.'

They are correct in their assertion about a triphasic pattern; but if by 'RBBB pattern' these authors mean, as many do, any pattern with a wide dominant positive wave in V1 then their statement is incorrect and the facts are: 1) statistically a monophasic (R) or diphasic (qR) pattern is more likely to represent ectopy than aberration; and 2) if such a complex peaks early (taller left than right 'rabbit-ear') the odds are strongly (at least $80 \%$ ) in favour of ventricular ectopy.

4) '. . the proven diagnosis of ventricular tachycardia.'

Their diagnosis was not by any means proven by their 'simple approach'; their manoeuvre, by producing retrograde VA block and eliminating $P$ waves, excluded an atrial but not junctional origin of the tachycardia and was no more diagnosistic than would be the demonstration of independent atrial activity.

In fact, the most compelling evidence in favour of ventricular tachycardia in this case is morphological: the QRS complexes are predominantly negative across the praecordium. This is a simpler approach (requiring only a glance at a 12-lead tracing) and a more convincing clue than the still equivocal evidence the authors have acquired by dint of atrial invasion plus vagal stimulation -procedures that are usually unnecessary and not without risk in the presence of acute infarction and an irritable myocardium.

The most important point is that the reader of Evans's article should not be beguiled into believing that an inadequate clue, sought if necessary by invasive means, is the primary target in the diagnosis of ventricular tachycardia.

To forestall the possible riposte that I too have been guilty of emphasizing the diagnostic value of independent atrial activity, let me hasten to acknowledge that in 1963-12 years ago when neither I nor anyone else knew any better-I stated that independent $P$ waves were of prime importance in the diagnosis of ventricular tachycardia (Marriott, 1963). But, during the past decade, a wealth of knowledge has accumulated about QRS morphology-triphasicity in V1 and V6, 'rabbit ears' in V1, rS or QS in V6, concordant praecordial positivity and negativity, bizarre frontal plane axis, wide $r$ in V1, etc. - and reliance on independent atrial activity is now a relic of former less enlightened times. Morphological clues are not always present; but when they are they afford much more diagnostic evidence than independent atrial activity.

Henry J. L. Marriott, St. Anthony's Hospital, Rogers Heart Foundation, St. Petersburg, Florida, U.S.A. 


\section{Reference}

Marriott, H. J. L. (1963). Armchair Arrhythmias. Tampa Tracings, Cardiology Center, Tampa General Hospital, Tampa, Florida.

Sir :

Dr. Marriott criticizes our statement that the 'Diagnosis of ventricular tachycardia is primarily dependent upon the demonstration of dissociated atrial and ventricular activity'. He asserts that this is an inadequate clue, 'as scientifically acceptable as to assume that the woman on the man's arm is his wife'. This statement closely resembles one in an article entitled 'AV dissociation revisited' by Marriott and Menendez (1966). However, in 'Armchair arrhythmias' Marriott (1963) makes two different statements. Speaking on ventricular aberration, he says (p. 7), 'These common pitfalls are avoided by keeping in mind the golden rule in the diagnosis of arrhythmias in general, cherchez le $P$. Marriott goes on to say (p. 20) that, 'The clues to diagnosis (ventricular tachycardia) are provided by the signs of AV dissociation (independent beating of atria and ventricles). In the electrocardiogram, the dissociation can be recognized and the ventricular origin inferred only if independent $P$ waves are visible or "Dressler beats" are present.' These latter views of Marriott concur with our statement and the views of many other experts. Marriott's several other points all stress the significance of QRS morphology in the recognition of ventricular tachycardia. We share, and discussed in our article, his views on the value of the triphasic QRS (right larger than left rabbit ears) supporting aberrancy. The absence of this finding may indeed favour ventricular origin, but certainly is not diagnostic, and particularly in a patient with a healed anterior wall myocardial infarct and ventricular aneurysm one would not rely on praecordial QRS morphology for diagnosis.

Although our patient did not have an acute myocardial infarction, as Marriott inferred, we concur that invasiveness in an acute ischaemic situation is hazardous. However, the hazards of recording an intra-atrial electrocardiogram in an electrically safe fashion are not only trivial, they are far less risky than many of the stresses to which our myocardial infarct patients are subjected. On the other hand, to make the distinction between ventricular tachycardia with intact retrograde conduction and junctional tachycardia with aberrancy and retrograde conduction requires high-risk ventricular invasion for His bundle recordings. The wiser part of valour when confronted with this differential, with or without the setting of an acute myocardial infarction, is to presume the more virulent process-ventricular tachycardia.

In summary, we are somewhat puzzled by Dr. Marriott's disparaging remarks regarding the value of $\mathbf{P}$ wave analysis. While we concur with his thoughts on the potential value of QRS morphology, we can think of several instances when this would be of little value or no value such as in our patient with an old anterior wall myocardial infarction and a healed ventricular aneurysm.

In actuality, in the clinical setting, the goal is to distinguish supraventricular tachycardia with aberrancy from either ventricular tachycardia or junctional tachycardia with aberrancy. In fact, junctional tachycardia with aberrancy is most often empirically treated as ventricular tachycardia unless immediate His bundle recordings can be obtained.

Despite Dr. Marriot's desire to shed his valuable contributions of the past, we still support them and remain imbued with the philosophy of Willie Sutton, a notorious Boston bank robber, who when asked why he robbed banks, replied: 'That's where the money is'. Analysis of the atrial electrical activity (cherchez le $P$ ) often detectable only in the atrium, is 'where the money is' in arrhythmia diagnosis.

\section{Gerald L. Evans, Framingham Union Hospital, 25 Evergreen St., Framingham, Mass., U.S.A.}

\section{References}

Marriott, H. J. L. (1963). Armchair arrhythmias. In Tampa Tracings. Cardiology Center, Tampa General Hospital, Tampa, Florida.

Marriott, H. J. L., and Menendez, M. M. (1966). AV dissociation revisited. Progress in Cardiovascular Diseases, 8, 522. 